\title{
Structural studies of the surface projections of Chlamydia trachomatis by electron microscopy
}

\author{
J.-J. CHANG, K. R. LEONARD* and Y.-X. ZHANG $\dagger$ \\ Institute of Biophysics, Academia Sinica, Beijing 100101, China, *European Molecular Biology Laboratory, \\ Postfach 10.2209, D-69012 Heidelberg, Germany and †Beijing Institute of Ophthalmology, Beijing, China
}

\begin{abstract}
Rod-like projections on the surface of Chlamydia trachomatis have been studied by a combination of computer image analysis and electron microscopy. The rods, c. 60-80 in diameter and c. $500 \AA$ in length, were found on the surface of prokaryocells of $C$. trachomatis inserted in the cytoplasmic membrane through a ring-like structure in the outer membrane. The rod-like structures were found at all stages of the life cycle, even in very small elementary bodies (EBs) of $C$. trachomatis and in vesicles $<0.2 \mu \mathrm{m}$. Computer image analysis of isolated rods indicated that they comprise helically arranged subunits with a periodicity of $c .50 \AA$. From their localisation and distribution, these structures may be related to the proliferation, or to the infectivity, of chlamydiae.
\end{abstract}

\section{Introduction}

Chlamydia trachomatis is a member of the genus Chlamydia which comprises obligate intracellular bacteria causing various diseases in both animals and man (reviewed in [1]). As with other chlamydia species, C. trachomatis can be distinguished functionally during its developmental cycle between two different cell types, namely the elementary body (EB) which is the infectious stage and the reticulate body (RB) which is non-infectious. EBs may exist as small cocci, c. $0.3 \mu \mathrm{m}$ in diameter; the RBs may be larger, $c$. $1 \mu \mathrm{m}$ in diameter.

The best identified feature of chlamydiae is the specific structure of their envelopes. Like other species, $C$. trachomatis has been shown to have a regular hexagonal lattice within the outer membrane of the cell envelope [2]. In negatively stained preparations of strain TE55 of C. trachomatis, folds and grooves on the surface of EBs were also observed [3].

Some reports also describe the presence of short sticklike structures attached to the outer surface of chlamydiae. For example, projections on the surface of $C$. psittaci were found after thin-sectioning and staining with ruthenium red by Matsumoto [4], who

Received 21 Feb. 1997; revised version accepted 13 May 1997.

Corresponding author: Dr K. R. Leonard.

$\dagger$ Present address: Maxwell Finland Laboratory for Infectious Diseases, Boston Medical Center, Boston University School of Medicine, Boston, MA, USA. suggested that they were cytoplasmic-membrane sites where the projections were connected to DNA molecules. By negative staining and freeze-fracturing, the presence of projections on the surface of $C$. psittaci was confirmed $[5,6]$ and the surface projections were found not only in EBs but also in RBs. Each elementary body had 18-23 projections. Stick-like protuberances were also described [2].

Nichols et al. [7] observed two kinds of surface projections, spike-like rods and hemispheric projections in $C$. trachomatis by use of thin-sectioning techniques. Again, when Miyashita et al. [8] compared isolated $C$. pneumoniae TWAR, $C$. trachomatis L3/ 434/Bu, C. psittaci Call 10 and C. pneumoniae YK-41, they found that all strains possessed very similar morphological features, including surface projections. Matsumoto [9], in a preparation of isolated surface projections of $C$. trachomatis, reported that stain penetration occasionally outlined fine subunits and a central channel in the projections, suggesting that they are tubular with a helical arrangement of subunits. Although it has been proposed that the surface projections are related to multiplication of chlamydiae, their function, whether to anchor the parasites to host cells or to play a role in nutrient absorption, is still unknown (reviewed in [10]).

This paper describes the structure of the surface projections of $C$. trachomatis, studied in more detail by combining electron microscopy with computer image analysis. Finally, some possibilities of their functions related to proliferation and infection are discussed. 


\section{Materials and methods}

\section{Cultivation and purification of $C$. trachomatis}

Prokaryotic cells of $C$. trachomatis strain TE55 were cultured in yolk sac membranes [11] and the cultured cells were purified [3] as described previously. To visualise the hexagonal lattice on the envelopes as an identifying characteristic of $C$. trachomatis, some purified chlamydia cells were digested in sodium dodecyl sulphate $1 \% \mathrm{w} / \mathrm{v}$ or in sodium desoxycholate $1 \% \mathrm{w} / \mathrm{v}$ in phosphate-buffered saline (PBS, $\mathrm{pH} 7.2$ ). Some cells were also disrupted with a microprobeultrasonicator to break the envelopes into single layers.

\section{Electron microscopy}

Negatively stained specimens were prepared as follows: c. $5 \mu \mathrm{l}$ of chlamydial suspension was applied to hydrophilic carbon-coated grids freshly glow-discharged in air. After adsorption for $1 \mathrm{~min}$, a drop of phosphotungstic acid (PTA, 1\% w/v, pH 7.2) was added. Excess liquid was removed with filter paper and grids were dried in air.

Freeze-dried shadow-casting samples were prepared as follows: after removal of liquid with filter paper, grids with chlamydiae were frozen immediately in liquid
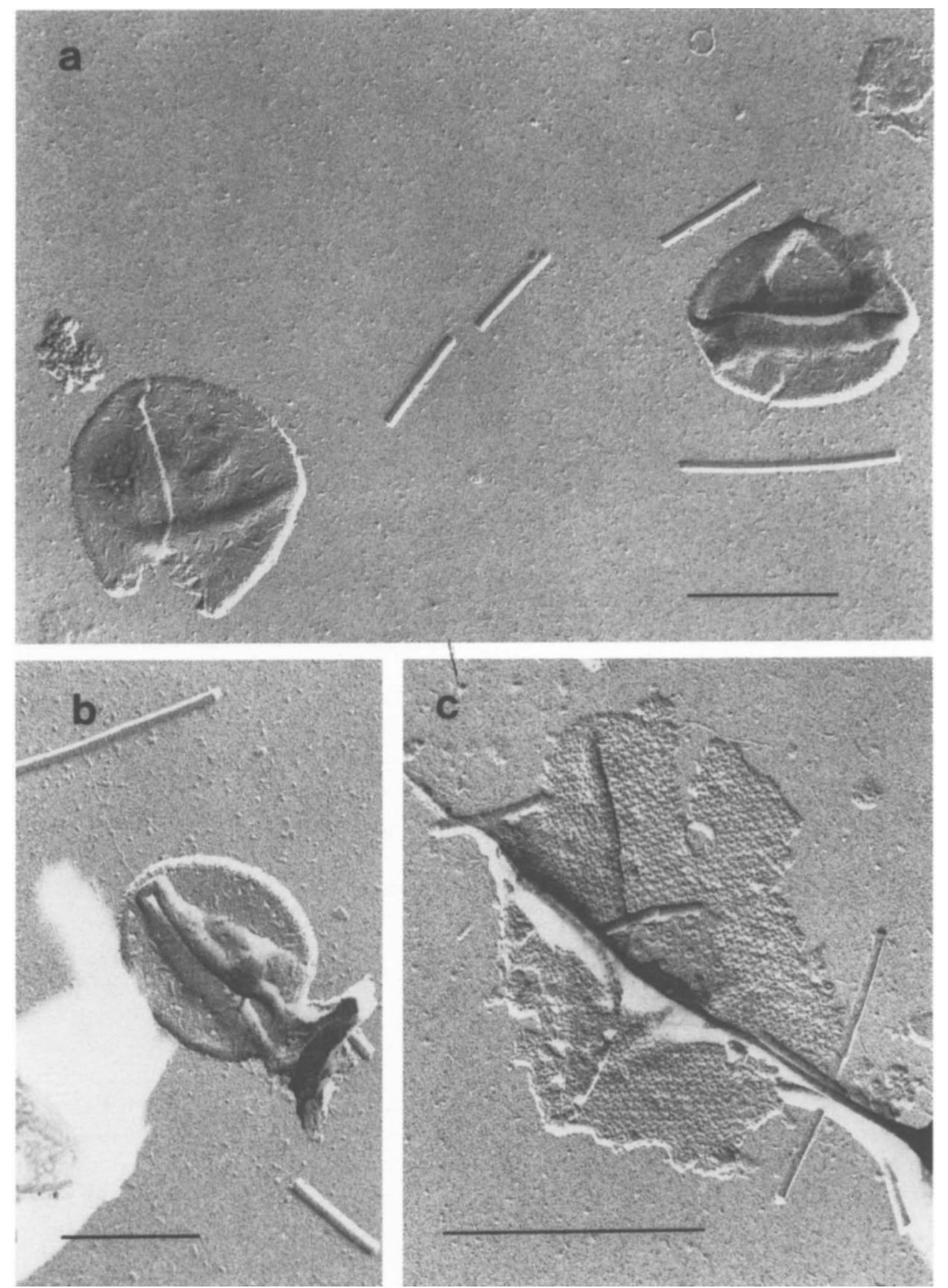

Fig. 1. Shadow-cast preparations of $C$. trachomatis mixed with TMV. a, b, small rod-like projections lie flat on the outer surface of intact cells; $\mathbf{c}$, the cell has broken open to reveal the regular inner lattice of the outer membrane. Scale bars, $0.5 \mu \mathrm{m}$. 
nitrogen and transferred to a cryochamber (Reichert Cryofract) under liquid nitrogen. Grids, after freezedrying in the cryochamber, were shadowed with platinum at an angle of $25^{\circ}$ in an evaporator (Balzers TCP-121). As an internal size standard, tobacco mosaic virus (TMV) was mixed with chlamydiae before being applying to grids.

Micrographs were taken in an electron microscope (Philips EM 400) operating at $80 \mathrm{kV}$ and at a magnification of $\times 28000$ with a low dose unit (Philips) for off-specimen focusing and astigmatism correction.

\section{Computer image analysis}

Images were digitised at $7 \mu \mathrm{m}$ raster on a scanning system (Zeiss Phodis/SCAI). The scanned pixel size was $4 \AA$. Fast Fourier transforms were calculated, after boxing and floating image data, by use of $1024 \times 1024$ arrays.

\section{Results}

\section{Distribution of projections}

Images of platinum-shadowed cells and broken envelopes are shown in Fig. 1. The hexagonal lattice is
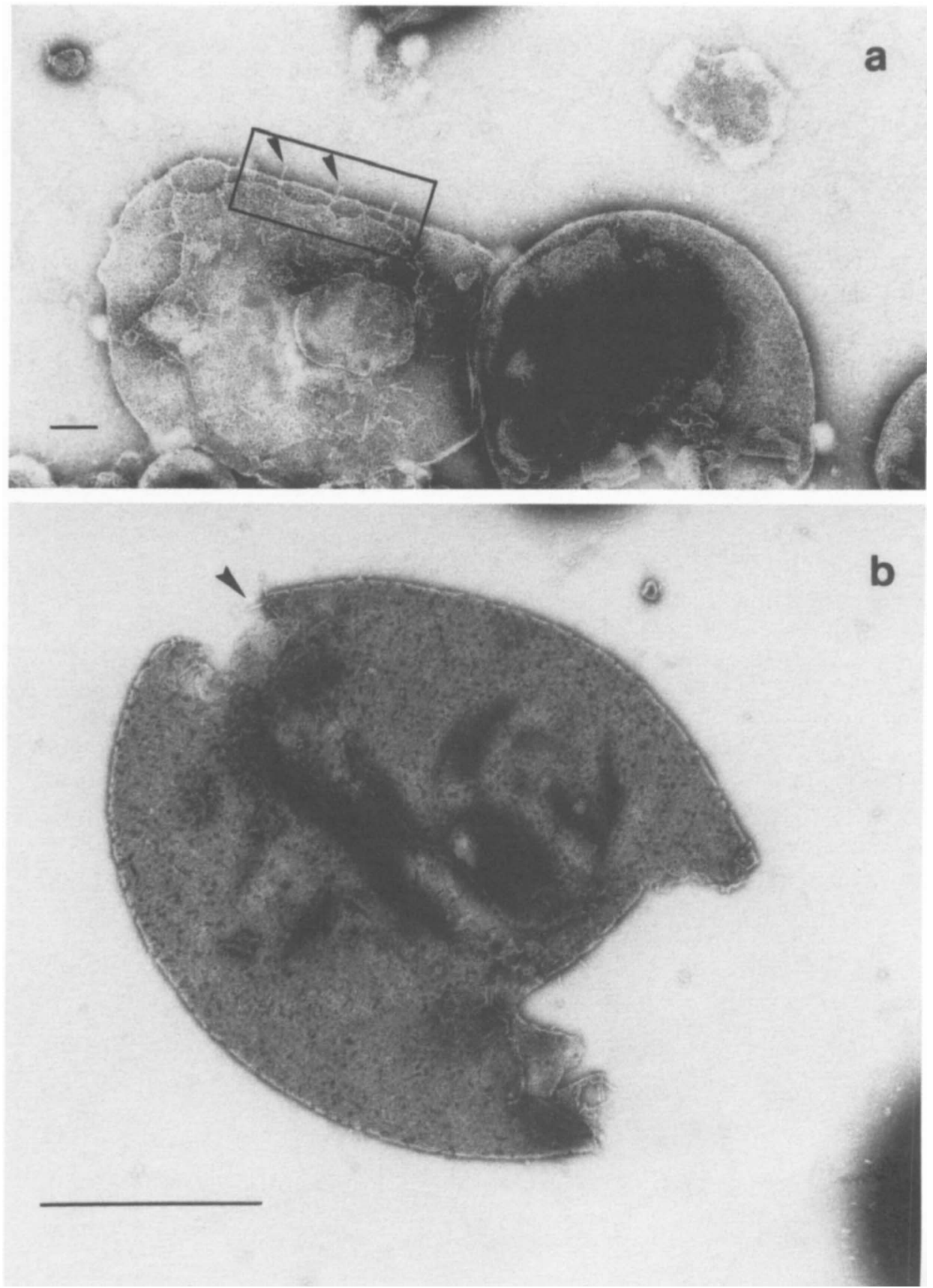

Fig. 2. Negatively stained intact EBs (a) and RB (b) showing rods inserting into the surface of the prokaryotic cells (arrowed). a, a region containing several rods which traverse the two cell membranes is outlined by a box (shown at higher magnification in Fig. 6). Scale bars a, $100 \mathrm{~nm}, \mathrm{~b}, 0.5 \mu \mathrm{m}$. 
clearly visible on the inner surface of a broken envelope. The outer surface of intact cells is smoother in appearance, with small rods lying irregularly over the envelope. Images of negatively stained intact EB and RB are shown in Fig. 2. In each case, surface projections or rods are indicated at the edge of cells. In addition, rods in profile are shown lying on the surface of one cell (Fig. 2a).

\section{Structure of surface projections}

A negatively stained sample of disrupted envelopes with rod-like projections broken away from the cell envelopes is shown in Fig. 3. The hexagonal cell envelope lattice is clearly visible, as are several ringlike structures superimposed irregularly on the lattice. The rings are $c .150 \AA$ in width, with a central stainfilled hole $c$. $80-100 \AA$ in diameter.

Selected images of negatively stained fragments of rods are presented in Fig. 4. A fine stain-filled channel is just visible along the centre of the rods (Fig. $4 a, b$ ). A full-length rod, $c$. $500 \AA$ in length and $70 \AA$ in diameter, is shown in Fig. $4 f$, with a computercalculated diffraction pattern from that particle shown in Fig. 4g. The diffraction patterns from 11 samples

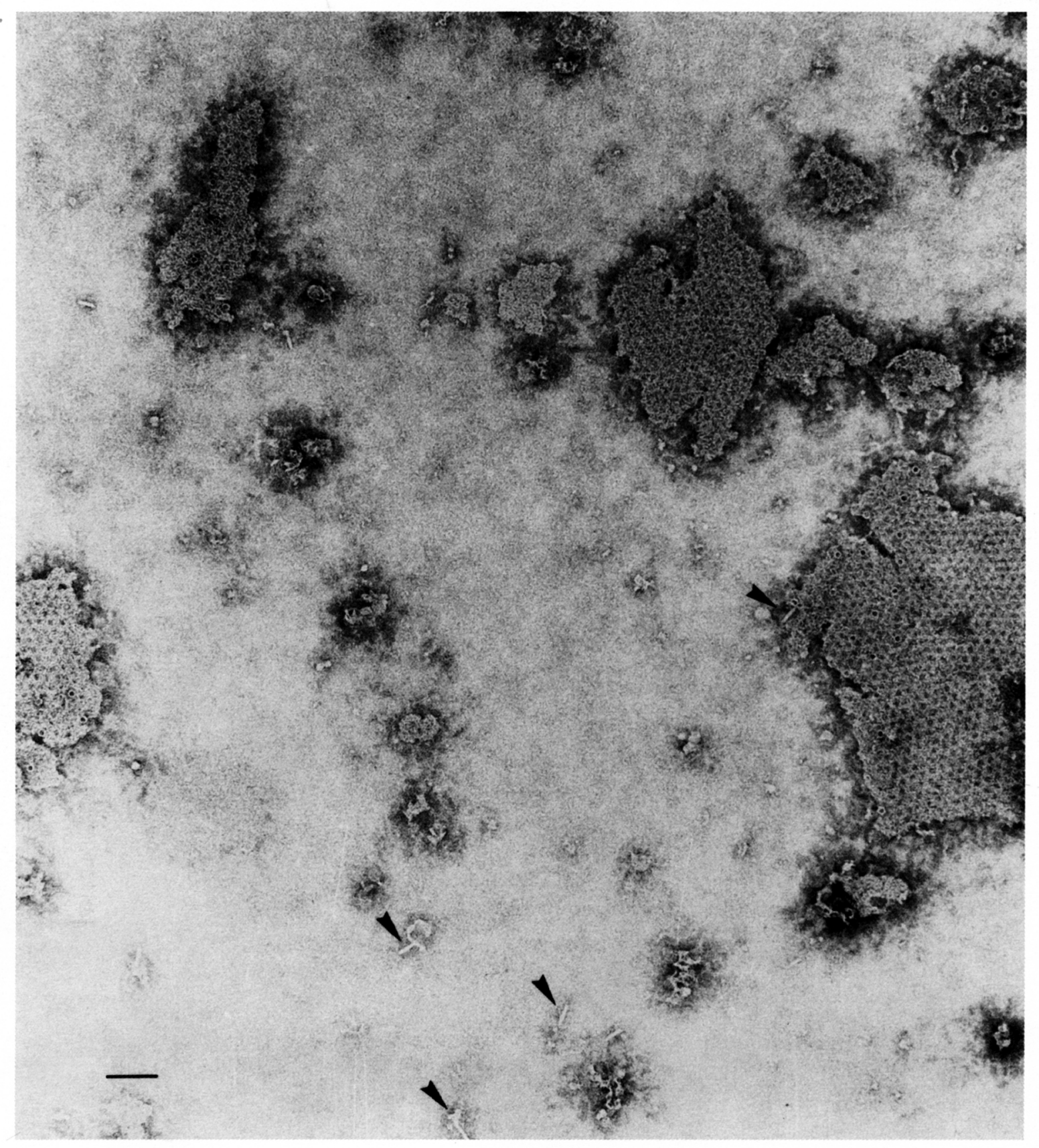

Fig. 3. Image of negatively stained patches of broken cells after SDS treatment and ultrasonication showing the hexagonal lattice of the outer membrane and a number of ring-like structures distributed randomly across the patches. Broken rods at the edges of the patches and in the background are arrowed. Scale bar, $0.5 \mu \mathrm{m}$. 

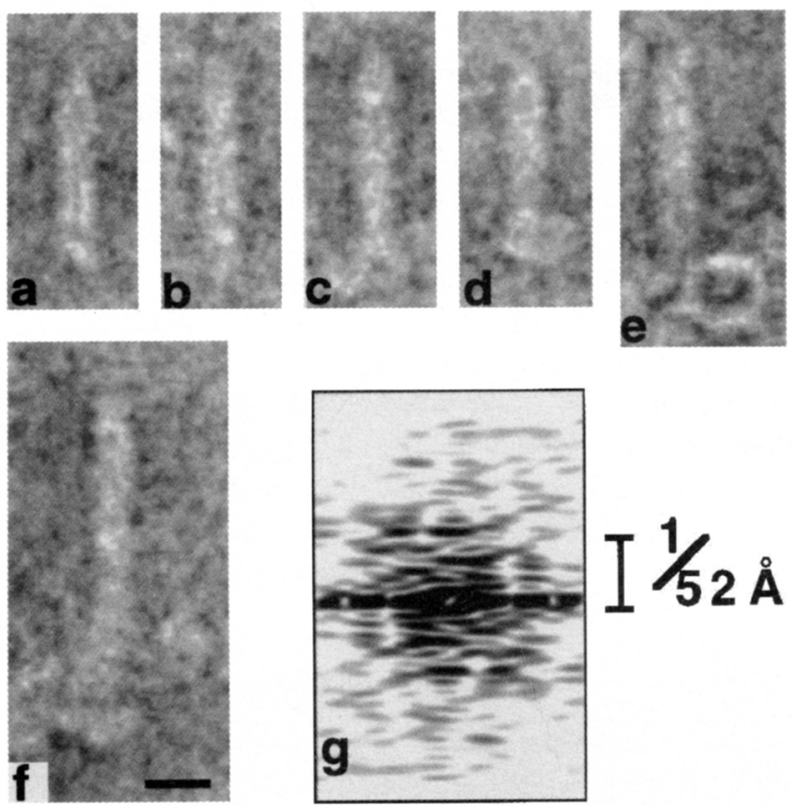

Fig. 4. Isolated projections after SDS treatment and ultrasonication. Broken fragments c. $200 \AA$ in length are shown $(\mathbf{a}-\mathbf{e})$. A ring-like structure is visible at the base of the rod (e). A full length $\operatorname{rod}(c .400 \AA$ ) is shown in (f) from which the computer-calculated diffraction pattern (shown in $\mathbf{g}$ ) has been obtained. Two layer lines at c. $50 \AA$ and $100 \AA$ reciprocal spacing are visible (g). Scale bar, a-f, $100 \AA$.

were weak, as only very short lengths of particle could be selected. In all cases, a layer line was visible at about $50 \pm 4 \AA$. A second layer line at c. $100 \AA$ is also visible (Fig. $4 \mathrm{~g}$ ).

A simple model to explain this result is shown (Fig. 5 ), taking into account the diameter of the rods and the presence of two main helical directions separating the subunits. A radial projection of the surface lattice of a simple helix, with a radius of $35 \AA$, and two principal helical directions corresponding to the $50 \AA$ and $100 \AA$ layer lines are shown in Fig. 5b. This model has $c$. six subunits per $100 \AA$ length. A solid

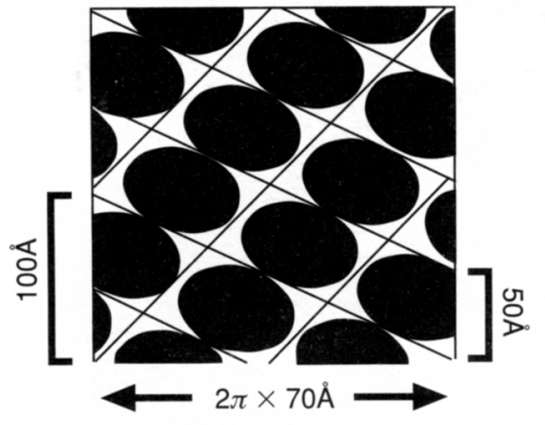

(a)

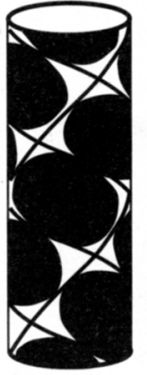

(b)
Fig. 5. Radial projection of a $70 \AA$ diameter helix with two principal helical lines of pitches $50 \AA$ and $100 \AA$ defining the surface lattice (a), corresponding to the layer lines seen in Fig. 4g. Schematic view of this lattice on the surface of the rod is shown (b). cylinder of protein with $35 \mathrm{~A}$ radius would correspond to a volume of $385000 \AA^{3} / 100 \AA$ length. Taking the density of protein to be $0.8 \mathrm{Da} / \AA^{3}$, this would give a mass of c. $51 \mathrm{kDa}$ per subunit. Allowing a $7 \AA$ radius channel at the centre of the rod, as suggested by the penetration of stain, the subunit mass would be reduced to c. $50 \mathrm{kDa}$.

\section{Localisation of rod-like surface projections}

A ring-like structure is seen at the end of a broken rod (Fig. 4e), with a central hole of the same diameter as the width of the rod. In size and shape, this ring corresponds to those seen in disrupted envelopes (Fig. 3). Fig. 6 shows images of rods (enlarged from the boxed area shown in Fig. 2a). These negatively stained samples show two cell membranes in profile. There appears to be a 'collar' around the rod, just below the inner face of the outer membrane (Fig. 6b, c). Taken together, these observations suggest that the ring or collar structures are present at the point where the rods pass through the outer membrane. The rods traverse the inter-membrane space and extend through the outer membrane. As may be seen, the distance between the two membranes varies; thus, the rods appear to be strongly anchored to the inner membrane but are loosely attached as they pass through the outer membrane.
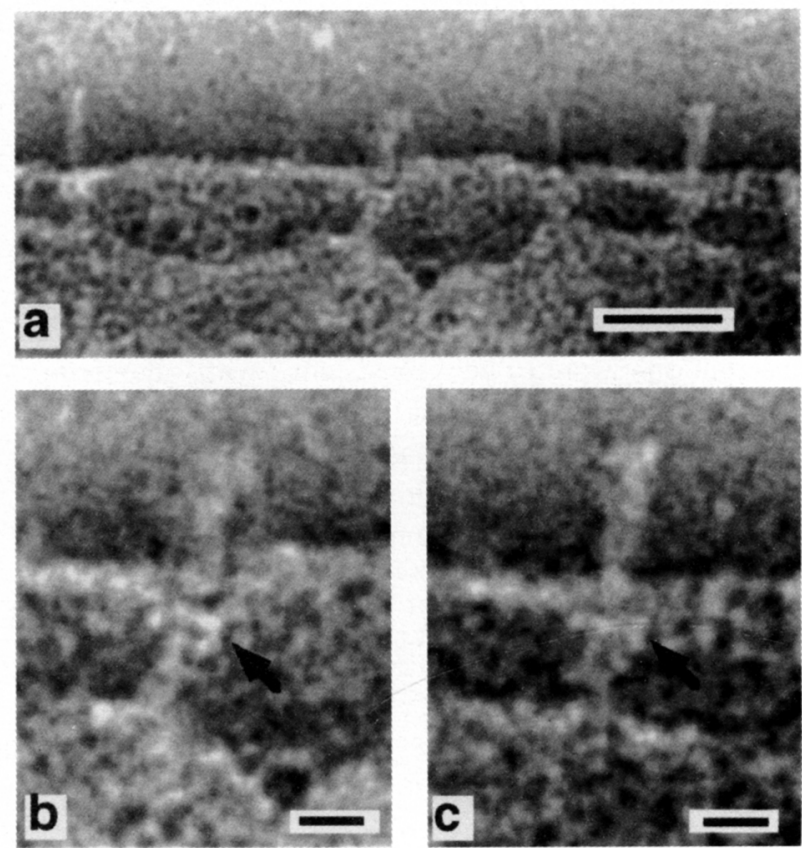

Fig. 6. Computer-scanned and enlarged images from the boxed area in Fig. 2. Negative stain has penetrated the region between the inner and outer membranes. Rods are anchored to the inner plasma membrane at one end and pass through the outer membrane. Just below the outer membrane a structure is visible (arrowed) which appears to be attached to the rod. The width of this structure is the same as that of the ring seen in Fig. $4 \mathrm{e}$ and may be a collar surrounding the rod. The length of rod between the inner and outer membranes is variable, suggesting that rods can pull through this collar. Scale bar a, $0.5 \mu \mathrm{m} ; \mathrm{b}$, c. $10 \mathrm{~nm}$. 


\section{Discussion}

The results of the present study support a model in which the rod-like projections on the surface of $C$. trachomatis are helical tubes with a narrow, almost invisible, central channel. The overall length of the rod is c. $500 \AA$. One end is anchored to the inner membrane with the remainder passing relatively freely through the outer membrane, such that a segment $(c$. $300 \AA$ ) projects from the surface. Broken rods found after ultrasonication are usually c. $300 \AA$ in length, suggesting that they have sheared off at the level of the outer membrane. These dimensions are similar to those found for C. psittaci [5], but less than those reported for C. trachomatis [7].

The approximate estimate of $50 \mathrm{kDa}$ for the size of the protein subunit making up the surface projections is based on a simple model which does not take into account the shape of the subunit. At present no direct data (e.g., measurements in mol.wt from SDS gels) exist for the subunit, which is a very minor component of the outer membrane. However, it is possible that a protein of this size, which should be present in both EB and RB stages, may be antigenic or may be available for surface labelling by chemical methods. Two possible candidates are the antigenic 59-kDa protein present in both $\mathrm{EB}$ and $\mathrm{RB}$ [12] or the $38-\mathrm{kDa}$ cytadhesin protein [13] shown to be present on the surface of EBs.

The distribution of rods varied on the cell surface among different cells as well as on different areas of the same cell surface. The number and distribution of ring-like structures seen in images of the hexagonal inner surface of the outer membrane was similar to that seen for the rods on the outer surface. This observation would be consistent with the rings forming an opening in the outer membrane through which the rods might pass relatively freely. The function of the rod-like projections remains unclear. The stain-filled central channel of the rod that appears faintly in some images, is probably $<15 \AA$ in diameter. Unless this is a result of shrinkage during specimen preparation, the channel would be too small for DNA or protein to pass through. However, it may be an ion channel. Alternatively, the rods may be involved in binding to target cells or in transmitting signals to the inner (cytoplasmic) membrane on binding. However, they are found at all developmental stages, even in the very small EBs, suggesting that their presence is necessary for the proliferation of chlamydial cells or to contribute to the infectivity of these obligate intracellular bacteria.

\section{References}

1. Ward ME. The immunobiology and immunopathology of chlamydial infections. APMIS 1995; 103: 769-796.

2. Chang J-J, Leonard K, Arad T, Pitt T, Zhang ZX, Zhang LH. Structural studies of the outer envelope of Chlamydia trachomatis by electron microscopy. I Mol Biol 1982; 161: 579-590.

3. Zhang ZX, Meng XM, Zhang LH, Su H, Li RD. Studies on the ultrastructure of envelope of elementary bodies of Chlamydia trachomatis. Sci China B 1980; 23: 1208-1217.

4. Matsumoto A. Electron microscopic observations of surface projections and related intercellular structures of Chlamydia organisms. J Electron Microsc 1981; 30: 315-320.

5. Matsumoto A. Electron microscopic observations of surface projections of Chlamydia psittaci reticulate bodies. $J$ Bacteriol 1982; 150: 358-364.

6. Matsumoto A. Surface projections of Chlamydia psittaci elementary bodies as revealed by freeze-deep-etching. $J$ Bacteriol 1982; 151: 1040-1042.

7. Nichols BA, Setzer PY, Pang F, Dawson CR. New view of the surface projections of Chlamydia trachomatis. $J$ Bacteriol 1985; 164: 344-349.

8. Miyashita N, Kanamoto Y, Matsumoto A. The morphology of Chlamydia pneumoniae. J Med Microbiol 1993; 38: 418-425.

9. Matsumoto A. Structural characteristics of Chlamydial bodies. In: Barron AL (ed) Microbiology of chlamydia. Boca Raton, FL, CRC Press. 1988: 21-45.

10. Raulston JE. Chlamydial envelope components and pathogenhost cell interactions. Mol Microbiol 1995; 15: 607-616.

11. Tang F-F, Chang H-L, Huang Y-T, Wang K-C. Studies on the etiology of trachoma with special reference to isolation of the virus in chick embryo. Chin Med $J$ 1957; 75: 429-447.

12. Kahane S, Weinstein Y, Sarov I. Cloning, characterization and sequence of a novel $59-\mathrm{kDa}$ protein of Chlamydia trachomatis. Gene 1990; 90: 61-67.

13. Joseph TD, Bose SK. A heat-labile protein of Chlamydia trachomatis binds to HeLa cells and inhibits the adherence of chlamydiae. Proc Natl Acad Sci USA 1991; 88: 4054-4058. 